\title{
System for remote configuration and over the air updates in restricted environments
}

\author{
Marko Zabreznik \\ University of Maribor \\ Faculty of Electrical Engineering \\ and Computer Science \\ Koroška cesta 46, Maribor \\ marko.zabreznik@student.um.si
}

\author{
Jernej Kranjec \\ University of Maribor \\ Faculty of Electrical Engineering \\ and Computer Science \\ Koroška cesta 46, Maribor \\ jernej.kranjec@um.si
}

\begin{abstract}
This paper illustrates a system for configuring, updating, command execution, and data retrieval via limited communication links of restricted embedded real-time operating system. A custom domain-specific language design and reference implementation are proposed, which would simplify the creation of custom program tasks while keeping data transfers low and facilitating differential updates to any software component. The proposed implementation extends the FreeRTOS real-time operating system running on an ARMbased microcontroller and connects to a remote command server via a limited network connection. External factors such as power shortage, component failure, and connection loss are anticipated and handled by preset priority-based scenarios.
\end{abstract}

\section{Keywords}

real time operating system, remote control, domain specific language for remote task execution, data structures, remote sensing

\section{INTRODUCTION}

In recent years, the cost and accessibility of world-wide communication channels, low cost of sensor equipment, and accessible computer modules have given researchers new sources of data acquisition. Systems used for such applications need to operate autonomously, are remote or completely inaccessible while also potentially under limited power, intermittent network connection, harsh or unpredictable weather, and other environmental hazards. Depending on those conditions, the objective or priorities might change during the lifetime of the mission.

The goal of this paper is to introduce a solution for embedded systems that require executing multiple different tasks (e.g., collecting data from various sensors, data processing, information storage and transmission), autonomous control over task execution based on external parameters (e.g., available power or sensor activity), remote configuration, and software updates. Presented solution would provide for more straightforward creation of such systems as it would allow for modular hardware components to be assembled into various configurations while only require to produce missing software from module templates, the behavior of which is controlled by a known set of parameters. To accomplish this, we propose a domain-specific language extension for a widely supported real-time operating system FreeRTOS to define scenarios that have associated tasks, conditions, and priorities. The same scenario definition is also used to partition the software into blocks, allowing for over-the-air updates and the addition of new scenarios remotely. Furthermore, the scenario definition is also used to prioritize the limited connection and system resources, and to allow for direct control.

\section{PROPOSED DESIGN}

The proposed design incorporates many dynamic components and thus vulnerable to corrupt, misconfigured, or buggy software. For that reason, the software is divided into a safe, minimal system (Bootstrap), and the schedule based configurable (Operating System) with the Meta-Scheduler (see Figure 1).

Both the Bootstrap and the Operating System can understand the basic programming instructions that work in all cases. Once an error in execution or corruption of the software is detected and can not be recovered from, the microcontroller reboots into the safe Bootstrap mode sends out a distress signal and waits for commands.

\subsection{Scenario}

A Scenario (see Figure 1) is the basic unit of the design that is independent of any other generic task, carries its configuration, and takes up any predefined blocks on the flash. The scenario can define a schedule and period of execution, the priority it needs, and the power it requires. We use those parameters to decide when and if the task is to run. The parameters are defined using a domain-specific language at compile-time but can change in the runtime with configuration stored in the microcontroller flash for each scenario.

\subsubsection{Settings}

The settings parameter defines the size of the binary blob the scenario can use for configuration data and is generally 


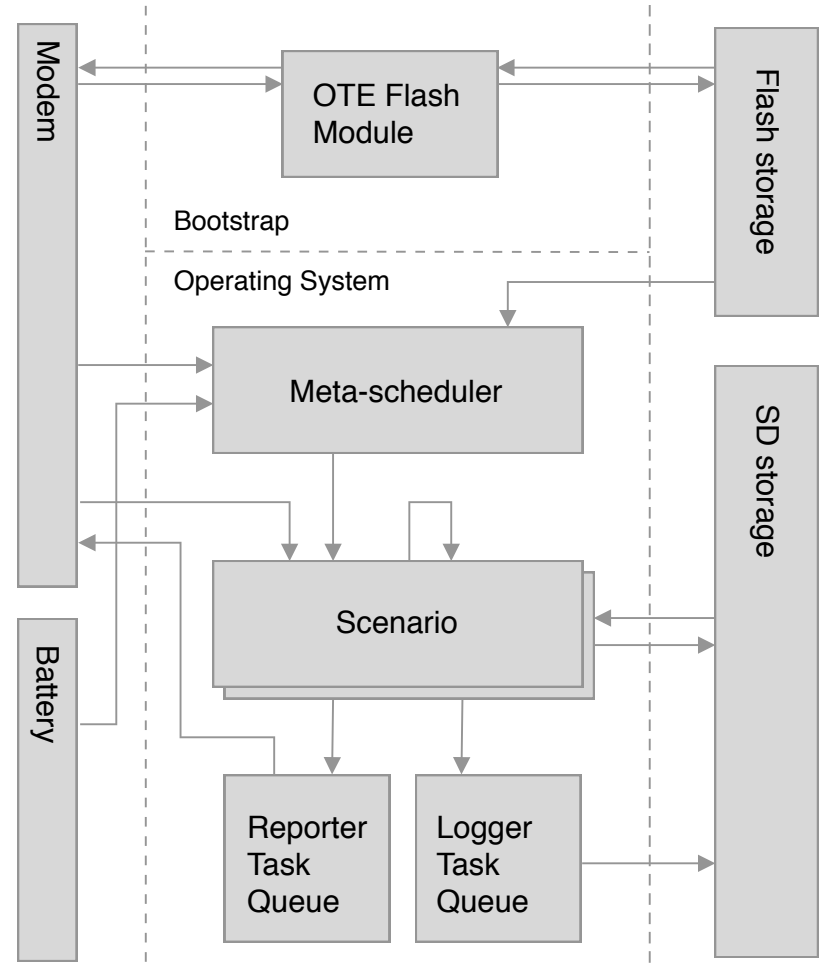

Figure 1: Overview of main logical software components and example hardware modules

only read from the scenario it belongs to. The settings are stored in the microcontroller flash and are accessed when a task requires them. The action of writing settings is done only via server commands.

\subsubsection{Schedule and period}

The basis for the schedule is time intervals in which the scenario runs and repetitions that happen in those defined intervals. The Meta-Scheduler uses the configuration to wake up the tasks at the specified hours and gives the task a timeout that co-responds with the set period. In this way, we can run the meta-scheduler in periods not more than one hour. The meta-scheduler is idempotent, so running it multiple consecutive times will not affect the running tasks.

\subsubsection{Priorities}

Priorities are the way the Meta-Scheduler decides if a task should run at all, or if it should only run when there is enough power in the budget or always in the case of critical tasks. The report priority is used for reporting and is there to keep a budget on power availability and network traffic.

\subsubsection{Power}

The power setting is a way to tell the Meta-Scheduler how much power a task needs to be able to run so that significant power-consuming tasks will only run when there is enough energy available. Specific scenarios can execute depending on power status. Configuration assumes the following conditions: the battery is full, the solar array produces power, the battery is charging, or when the battery is almost drained, but we still want the task to run regardless of any power

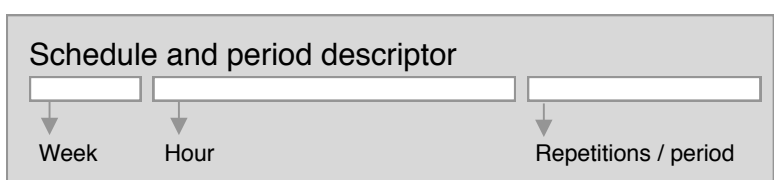

\begin{tabular}{|ll|}
\hline Priority & \\
\hline Run & Report \\
00 - off $\quad 01$ - when free & 00 - off $\quad 01-$ pad \\
$10-\mathrm{n} / \mathrm{a} \quad 11$ - always & 10 - force pad 11 - force \\
\hline
\end{tabular}

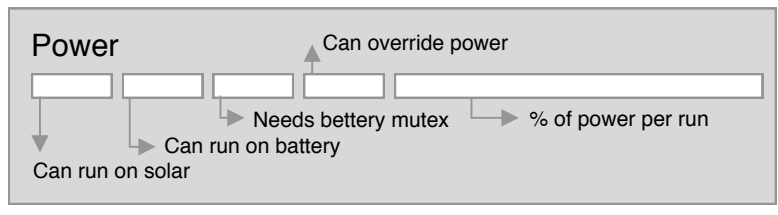

Settings pointer

Task handler

Last run
$\square$ Timestamp
$\square$ Repeats

Figure 2: Scenario configuration (read-only variables in gray and runtime variables in white blocks)

budgets. The power budget for a task is defined in the percentage of the total power available to the probe.

\subsubsection{Task Notifications}

A task is based on the FreeRTOS task [1] and its notification variable that has, by default 4 bytes. The first 3 bytes are used for custom messages send to the task, and the last byte is used as flags to set repeats (timeouts) that the task should run. Zero repeats mean the task will only run once in that time-frame and put themselves into infinite sleep mode after that. One or more repeats places the task into a timeout of 60 minutes divided by the repeat rate, at a minimum of 5-minute interval. More granular repeats can be made within the task itself and are not registered with the MetaScheduler. Any task with an interval will run until the next time the Meta-Scheduler is run, and it stops the execution.

\subsubsection{Logger}

The Logger is a special scenario, available to all tasks for logging purposes. It runs in a best-effort manner, using multiple queues with messages grouped by severity. The logs are handled with the notification variable of the header.

The logs can be optionally saved on the SD card and only if there is enough power and enough time has passed since the last write. Since the queue could have been filled up since the last write, the queue is cycled with the oldest messages being reused. In the event of a hardware failure, the Logger is disabled.

Each entry has a timestamp, a scenario id, and a fixed size data blob to store the log message or data. 


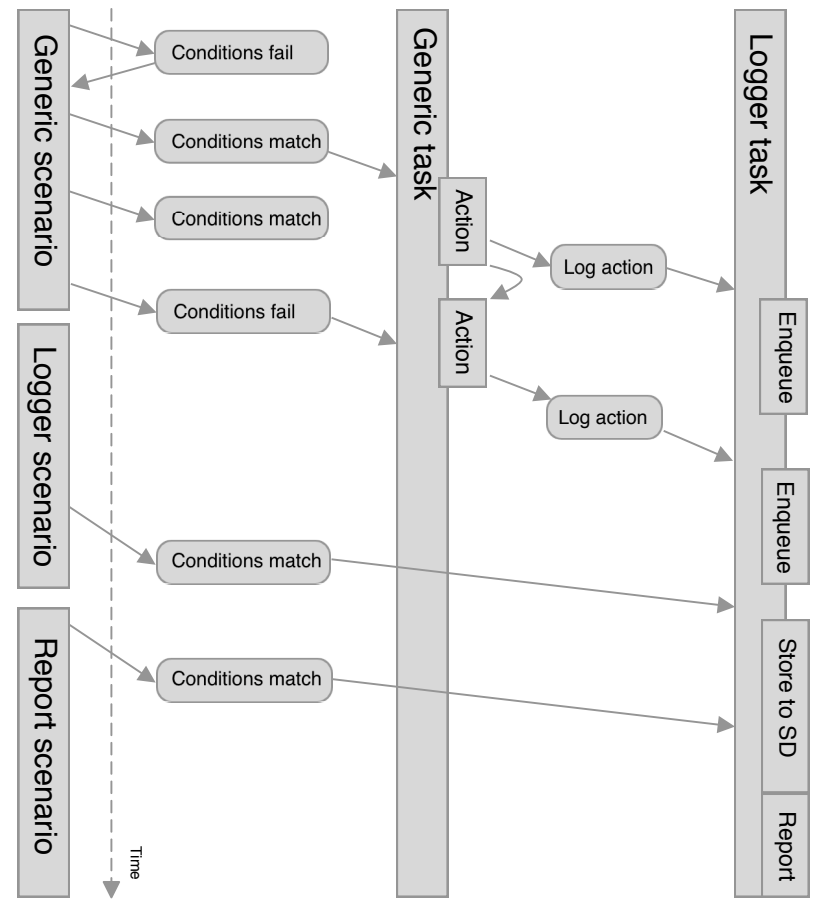

Figure 3: Timeline of an example generic task, logger, and reporter

\subsection{Communication}

Communication size is reduced to a minimum with compression based on common-knowledge. Since the server has the same template, we can use that to avoid sending headers before each data blob in some cases. The packet size, in our example, the Iridium satellite network, is limited to 360 bytes from the probe and 270 bytes to the probe with charge intervals of 50 bytes [2]. We use those limits to optimize expenses on low-value data.

\subsubsection{Reporting}

The reporter is a special scenario, available to all tasks for reporting purposes. Each priority level has a queue, and repeat entries are overwritten. Scenarios send notifications to the Reporter task using the notification variable and are saved to the queue, awaiting packet construction. At predefined intervals, the packet is constructed using the available reports (see figure 1). Critical reports can be sent and are flushed to the modem instantly using a special flag in the report command.

To save space, we encode the reports into several kinds of formats, denoted by the first few bits in the message.

\subsubsection{Templated Report}

The primary way to report is to use the section arrangement on the microcontroller flash and the priority values to order the scenarios. The first bit in the stream of each scenario reports if the bits that follow are from the scenario. The domain-specific language denotes the length of the data the scenario will report. If the report bit is zero, we skip to the next scenario with only 1 bit used to determine the scenario
Flash Command

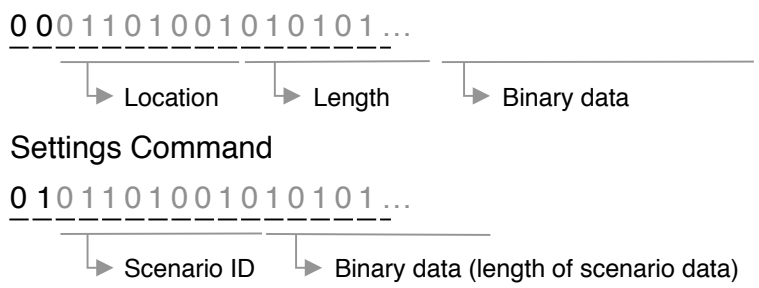

Control Command

$\frac{101}{\longrightarrow \text { Scenario ID }} \rightarrow$ Notification binary data (3 bytes)

Template report

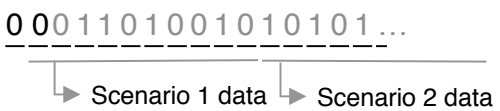

Key-value report

0 1011010010001101 ...

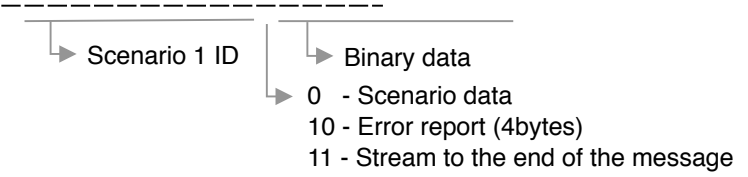

Custom report

101101001010101

Figure 4: Command and report encoding format

has nothing to report. This method will not apply if there are any scenarios with errors to report. If there is enough space at the end of the packet, we fill the rest of the 50-byte section with key-values, as explained in the next section.

\subsubsection{Key-Value Reporting}

The Key-Value is the most basic report used when there are errors or unknown data type in the templated report. The scenarios are again listed one after the other, prefixed with the scenario key and the type of data prefix. Unlike the templated report, the type of data can denote if the following bytes are fixed width or if a length follows.

\subsubsection{Custom Report}

The custom report is a special report that can be only sent on demand from a scenario and has no structure beyond the first bit. This type of report is intended for crash reports and critical errors and is not meant to be automatically handled.

\subsection{Commands}

Commands are packets sent from the server to configure or run scenarios and to update any part of the microcontroller flash.

\subsubsection{Flash Commands}


Flash commands (prefix 00) are simple write commands that can be used either in the Bootstrap or the full Operating System mode. The predefined nature of the flash allows us to overwrite any scenario and core software. The command starts with a location in the microcontroller flash, followed by the length of the data to be written and the binary data itself. The structure can repeat to the end of the packet.

\subsubsection{Settings Commands}

The settings command (prefix 01) is attached to a scenario and therefore used with the Scenario id to find the location of the settings block in microcontroller flash. The predefined length provides safety from overflows. This structure can repeat to the end of the packet.

\subsubsection{Control Commands}

Control commands are used to control task using their notification variable directly and can, in most cases, be only run within the full operating system mode. This structure is composed of 3 bytes that are available as parameters sent to the task.

\subsubsection{System Control Commands}

System control commands use the same pattern as simple control commands, but they use the predefined system namespace. These commands can be used in either Bootstrap or Operating System mode and are used for tasks like flashing, rebooting, and other non-scenario tasks.

\subsection{Over-the-air flash procedure}

Changing any part of the software, including the Bootstrap, system procedures, and scenarios can is accomplished with the flash procedure using system control commands.

The procedure should be started by resetting the mutable image on the flash storage with an exact copy of the original software in the microcontroller flash. The next steps are done using the flash commands to write changes into the mutable image. The last step is sending the flash system command with the hash of the image that we want to write into the boot storage.

If the prepared image hash does not match the provided value, a critical message is sent to the server, and the procedure is broken off. If the hash does match, the system is rebooted, the new image is written to the appropriate sector, and the bootstrap procedure started.

In the event of a critical failure, the server can send a command that flashes the original image to the appropriate location and repeats the bootstrap procedure. Alternatively, if desired, a complete custom image cand be sent at the expense of increased network data usage.

\subsection{Bootstrap}

Bootstrapping the system involves loading all the scenarios into memory. All the generic scenarios are stored in consecutive fixed width blocks with a header. The loader reads the header of each block for a magic number to see if the block contains a scenario and tests the checksum. If successful, the settings pointer is checked and if needed, the settings

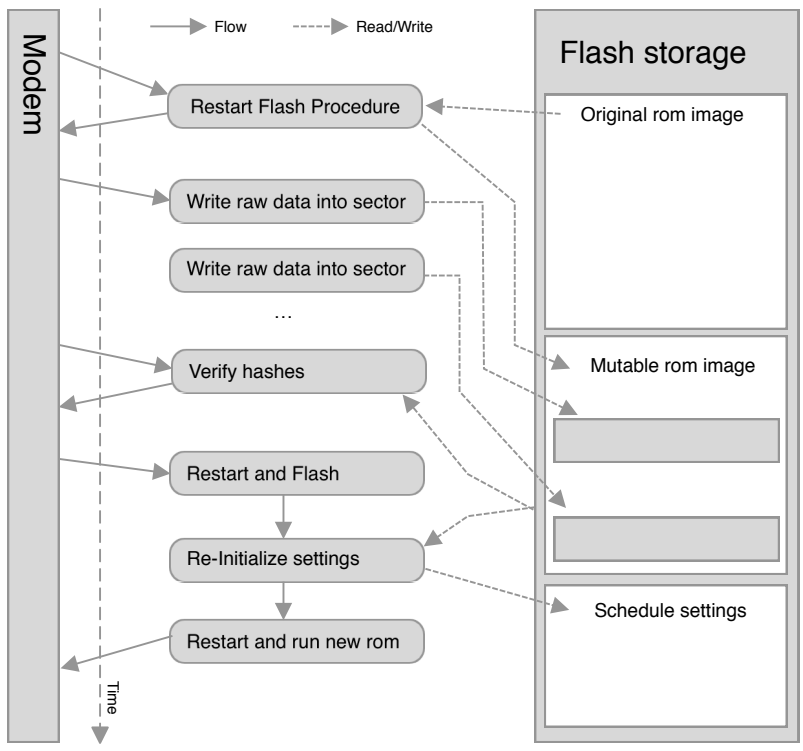

Figure 5: Timeline of a ota flash and re-initialization (left) with the flash layout (right)

block is initialized. Finally, the tasks of the scenario are run and placed into a infinite wait state.

\subsection{Conclusion}

The inspiration for designing such a system derives from a separate student project designing a floating sensor device, intended for data gathering in the ocean. As such, proposed implementation centers around the need for an embedded autonomous system with the primary goal of collecting data depending on detected conditions, available energy reserves, and network availability or cost. The lifetime of such a device is expected to exceed a year. Therefore the system also takes into account the need for remote over the air system updates and changing of execution parameters.

Current anticipated technical difficulties, regarding a working implementation on an actual microcontroller, revolves around physical memory or flash partitioning for remote updates, memory allocation and number of tasks being able to run or ques being able to exist as buffers and operating data storage, and the ability to correctly estimate the power available to the system for proper task execution.

\section{REFERENCES}

[1] R. Goyette. An analysis and description of the inner workings of the freertos kernel. Carleton University, 5, 2007.

[2] J. Hutcheson and M. Laurin. Network flexibility of the iridium (r) global mobile satellite system. 1995. 